\title{
Notificação de acidentes com exposição a material biológico: revisão integrativa
}

\section{Accidents notification with exposure to biological material: an integrative review}

Patrícia Dias Barreto ${ }^{1}$ Cristina Lavoyer Escudeiro²

\begin{abstract}
RESUMO
Objetiva-se identificar literaturas relacionadas à notificação de acidente de trabalho com exposição a material biológico. Revisão integrativa de artigos publicados em português, inglês e espanhol, nas bases MEDLINE,CINAHLe LILACS, utilizando os descritores: notificação, acidentes e eventos biológicos, exposição a agentes biológicos, e, sistemas de informação em saúde. Foram selecionadas 39 obras. Os acidentes de trabalho com exposição a material biológico conferem um risco significativo de contaminação aos profissionais de saúde, devendo ser adotadas medidas para minimizar os riscos de ocorrência do acidente. A exposição dos profissionais de saúde está diretamente ligada ao desenvolvimento de seus trabalhos e suas rotinas, associada, muitas vezes, com a ausência de uma conduta segura no ambiente de trabalho, uma vez que, no cotidiano, têm o contato e exposição ao sangue e fluidos corporais. Conclui-se que a notificação se apresenta como poderosa ferramenta para sinalizar, epidemiologicamente, os locais e agravos que estão emergindo no município do notificador; mostrando em que locais as ações de prevenção e promoção à saúde devem ser direcionadas, a que tipo de público e a quais agravos, especificamente.
\end{abstract}

Palavras-chave: Notificação; Acidentes e Eventos Biológicos; Exposição a Agentes Biológicos; Sistemas de Informação em Saúde.

\section{ABSTRACT}

The aim is to identify literature related to the notification of occupational accidents with exposure to biological material. Integrative review of articles published in Portuguese, English and Spanish, in the databases MEDLINE, CINAHL and LILACS, using the following descriptors: notification, accidents and biological events, exposure to biological agents, and information systems in health. 39 studies were selected. Occupational incidents with exposure to biological material confer a significant risk of contamination to professional's health and measures should be adopted to minimize the risk of occurrences of the accident. Exposure of professional's health is directly linked to the development of their work and their routines, associated often with the absence of a safe conduct in the workplace, as in everyday life have contact and exposure to blood and body fluids. It concludes that the notification is presented as a powerful tool to signal, epidemiologically, local and health problems that are emerging in the notifier's municipality; showing that local actions prevention and health promotion should be directed to what kind of public and which injuries specifically.

Keywords: Notification; Accidents and Biological Events; Exposure to Biological Agents; Information Systems in Health.

${ }^{1}$ Mestre em Enfermagem pelo programa MPEA-EEAAC da universidade Federal Fluminense, Coordenadora de enfermagem da Saúde Coletiva do Município de Araruama, Professora da Faculdade União Araruama de Ensino.

2 Doutora em Enfermagem, professora do Programa MPEA- EEAAC da Universidade Federal Fluminense, Coordenadora da Graduação em Enfermagem da Escola de Enfermagem Aurora de Afonso Costa. 


\section{INTRODUÇÃO}

A saúde é um bem resguardado na Constituição Federal como direito fundamental do cidadão. Segundo a Organização Mundial da Saúde (OMS), a saúde não se mostra apenas como ausência de doenças, e sim um conjunto de fatores que englobam o físico e o social. Portanto, tornase indispensável que o trabalhador de enfermagem goze de condições salubres e acompanhamento de sua saúde ocupacional. Fato que legalmente é garantido pela Portaria $n^{\circ} 1.823$ de 23 de agosto de 2012, onde é instituída a Politica Nacional do Trabalhado e da Trabalhadora. Esta enfatiza que cabe ao Sistema Único de Saúde (SUS) a execução das ações de saúde do trabalhador, e seu artigo $2^{\circ}$ definem os princípios e diretrizes a serem observados nas três esferas de gestão ${ }^{1}$.

Os profissionais de saúde, até a década de 80 não eram considerados como atores com potencial de risco de contaminação de doenças no exercício do trabalho, porém com o advento da epidemia de Imunodeficiência Humana Adquirida e conhecimento de suas formas de transmissão, tornou-se indispensável criar e aplicar medidas de proteção para os trabalhadores ${ }^{2}$.

A Política Nacional de Saúde do Trabalhador e Trabalhadora visa incorporar a categoria trabalho como determinante no processo saúde-doença do sujeito e coletividade, tendo como objetivos a identificação das necessidades, demandas e problemas de saúde dos trabalhadores no território, a realização da análise da situação de saúde dos trabalhadores, a intervenção nos processos e ambientais de trabalho e a produção de tecnologias de intervenção, avaliação e monitoramento da saúde dos trabalhadores ${ }^{3}$.

0 acidente de trabalho com material biológico ocorre quando o profissional, no manejo de suas atribuições, se vê exposto a sangue ou fluidos corporais. Tem-se como objeto desta revisão a notificação do acidente de trabalho por material biológico, apresentando importância no que tange ao monitoramento epidemiológico dos eventos, com a finalidade de sinalizar áreas ou locais onde estes eventos mais ocorrem. 0 monitoramento e medidas de proteção do trabalhador encontram-se amparadas no campo da saúde pública, pois existe um risco real de contaminação por patologias, como a transmissão por HIV, este com variação entre $0,3 \%$ e $0,1 \%{ }^{4}$.

Portanto, o estudo que ora se apresenta tem o objetivo de identificar literaturas relacionadas à notificação de acidente de trabalho com exposição a material biológico.

\section{MÉTODO}

Trata-se de uma revisão integrativa da literatura, que consiste em sintetizar múltiplos estudos publicados sobre determinado assunto e identificar lacunas que precisam ser preenchidas com a realização de novos estudos ${ }^{5}$.

A construção do estado da arte deu-se através de pesquisa nas bases de dados: Literatura Latino-Americana e do Caribe em Ciências da Saúde (LILACS), Medical Literature Analysis and Retrieval System Online (MEDLINE) e Cumulative Index to Nursing and Allied Health Literature (CINAHL), tendo como recorte temporal os anos de 2005 a 2015, e utilizando os descritores: notificação, acidentes e eventos biológicos, exposição a agentes biológicos, e sistemas de informação em saúde. Como critérios de inclusão considerou-se os artigos totalmente disponíveis; teses totalmente disponíveis, os trabalhos publicados nos idiomas português, inglês ou espanhol, cuja metodologia adotada permitisse obter evidências sobre o tema. Para os critérios de exclusão delimitou-se: estudos de revisão; fora do recorte temporal; estudos repetidos.

Foram encontrados, nas três bases pesquisadas, 1.577 artigos e 2 teses. Aplicados os critérios de inclusão e exclusão, analisou-se 39 obras, das quais 37 artigos e 2 teses, ficando assim distribuídas: 6 artigos na Cinahl, 5 artigos na Medline, e 26 artigos e 2 teses na Lilacs.

Quadro 1. Distribuição da literatura localizada e selecionada na Lilacs, Medline e Cinahl - 2005-2015

\begin{tabular}{|c|c|c|c|c|c|c|c|c|}
\hline \multirow[b]{2}{*}{ Descritores } & \multicolumn{2}{|c|}{ Lilacs } & \multicolumn{2}{|c|}{ Medline } & \multicolumn{2}{|c|}{ Cinahl } & \multicolumn{2}{|c|}{ Total } \\
\hline & $\mathbf{L}$ & $\mathbf{S}$ & $\mathbf{L}$ & $\mathbf{S}$ & $\mathbf{L}$ & $\mathbf{S}$ & $\mathbf{L}$ & $\mathbf{S}$ \\
\hline [notificação] and [acidentes e eventos biológicos] & 13 & 2 & 0 & 0 & 10 & 0 & 23 & 2 \\
\hline $\begin{array}{l}\text { [exposição a agentes biológicos] and [sistemas de } \\
\text { informação em saúde] }\end{array}$ & 28 & 1 & 37 & 3 & 52 & 3 & 117 & 7 \\
\hline $\begin{array}{l}\text { [acidentes e eventos biológicos] and [exposição a agentes } \\
\text { biológicos] }\end{array}$ & 21 & 7 & 367 & 1 & 127 & 1 & 515 & 9 \\
\hline [notificação] and [exposição a agentes biológicos] & 45 & 17 & 139 & 1 & 134 & 1 & 318 & 19 \\
\hline [notificação] and [sistemas de informação em saúde] & 13 & 1 & 285 & 0 & 290 & 0 & 588 & 1 \\
\hline $\begin{array}{l}\text { [acidentes e eventos biológicos] and [sistemas de } \\
\text { informação em saúde] }\end{array}$ & 0 & 0 & 0 & 0 & 18 & 1 & 18 & 1 \\
\hline Total & 120 & 28 & 828 & 5 & 631 & 6 & 1.579 & 39 \\
\hline
\end{tabular}

Fonte: elaboração dos autores, 2016.

Legenda: L- Literatura localizada

S - Literatura selecionada 
A estratégia "P: população/pacientes; I: intervenção; O: desfecho/outcome" (PIO) está delimitada como: P profissionais expostos a acidente biológico, I - Material Educativo Impresso para orientações sobre como realizar o preenchimento do formulário de notificação de acidente por material biológico, $\mathrm{O}$ - Realização do preenchimento do formulário de notificação de acidente por material biológico de maneira completa.

Foram encontrados na Lilacs 118 artigos e 2 teses, na qual foi utilizado o boleano and entre os descritores, realizando seis associações, resultando em 28 obras selecionadas para o estudo, das quais apenas uma era em idioma inglês e as demais em português.

A predominância das publicações selecionadas foram nos anos de 2014 (5 artigos) e 2010 (4 artigos); seguida dos anos de 2008, 2009, 2011 e 2012 com 3 artigos cada, sendo que em 2012 encontrou-se 2 artigos e 1 tese. Os anos de 2007, 2013 e 2015 tiveram 1 artigo. 0 ano de 2006 só apresentou 1 tese.

A busca de artigos na base Medline utilizando os Mesh,: notificação, acidentes e eventos biológicos, exposição a agentes biológicos, e sistemas de informação em saúde, com critérios de inclusão e exclusão, e o recorte temporal, localizou 833 artigos, sendo selecionados 5 artigos para análise.

Os cinco artigos selecionados nesta base encontram-se na língua inglesa, sendo 3 do ano de 2006, 1 de 2010 e 1 artigo do ano de 2011. Os artigos foram publicados em revistas científicas europeias, sendo os estudos realizados na Europa (3), na Arábia Saudita (1), e no Japão (1).

$\mathrm{Na}$ base Cinahl foram localizados 631 artigos, dos quais 6 atenderam aos critérios de inclusão e exclusão. Dos 6 artigos, 5 encontravam-se em língua portuguesa, e 1 em língua inglesa. Quanto aos anos de publicação 1 artigo era de 2009, 1 de 2010, 3 de 2011, e 1 de 2012.

Questão norteadora da pesquisa: Quais os recursos de informação utilizados para acompanhamento de acidentes ou exposições a agentes e materiais biológicos?

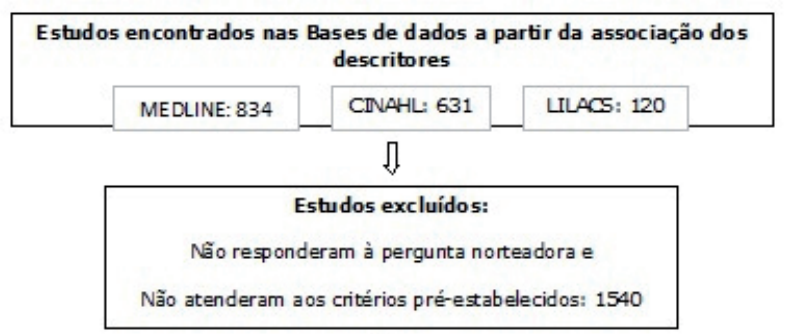

ป

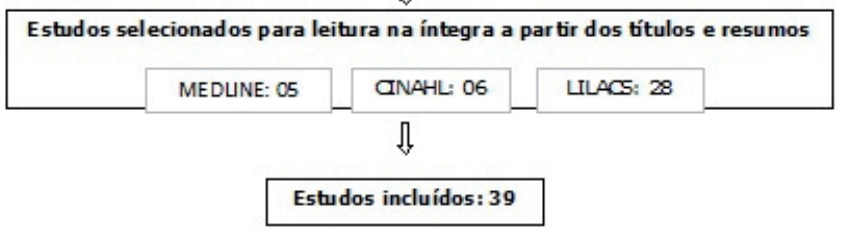

Figura 1- Fluxograma do Processo de Seleção dos Estudos Fonte: elaboração dos autores, 2016.

\section{RESULTADOS}

Dos artigos incluídos nesta revisão, observou-se o predomínio de publicações em revistas da área de enfermagem, sendo a sua maioria em periódicos nacionais. Em relação ao idioma, cinco são em língua estrangeira, os demais em português.

Os autores em sua maioria foram da área de enfermagem, não sendo possível delimitar a predominância em relação ao campo de estudo, estando presentes trabalhos que foram desenvolvidos em campos hospitalares e na atenção básica. Todos são originais, não sendo utilizadas revisões. 0 quantitativo dos artigos foi pesquisado e separado por anos, conforme quadro 2 a seguir.

Quadro 2. Distribuição de publicação por ano

\begin{tabular}{|c|c|}
\hline Ano publicação & Artigos ou tese \\
\hline 2006 & 4 \\
\hline 2007 & 2 \\
\hline 2008 & 3 \\
\hline 2009 & 4 \\
\hline 2010 & 6 \\
\hline 2011 & 7 \\
\hline 2012 & 4 \\
\hline 2013 & 3 \\
\hline 2014 & 4 \\
\hline 2015 & 2 \\
\hline
\end{tabular}

Fonte: elaboração dos autores, 2016.

Entre os artigos evidenciaram-se os cuidados voltados à promoção da saúde do trabalhador, inseridos na temática promoção e prevenção da saúde; estando os mais estudados na área da enfermagem, apresentando ainda um número menor de artigos da odontologia e medicina.

\section{DISCUSSÃO}

Com a finalidade de melhorar a interpretação, e discussão, os resultados foram divididos em dois eixos temáticos: 1- 0 acidente com material biológico; 2Condutas de prevenção e após o acidente de trabalho por material biológico:

\section{0 acidente por material biológico}

A exposição dos profissionais de saúde está diretamente ligada ao desenvolvimento de seu trabalho e sua rotina, associados muitas vezes com a ausência de uma conduta segura no ambiente de trabalho, uma vez que em seu cotidiano o contato e exposição ao sangue e fluidos corporais, contaminados ou não, são constituintes de sua rotina, estando assim, habitualmente expostos a doenças ocupacionais $s^{6-7}$. 
Define-se como trabalhador da saúde toda pessoa que desempenha atividade que envolva contato com pacientes, estando internados ou em laboratório ${ }^{8}$.

Neste contexto, ao desenvolver atividade produtiva no cuidado ao ser humano, quando por alguma eventualidade acidenta-se e entra em contato com sangue ou fluidos corporais, este trabalhador sofre um acidente de trabalho por material biológico, que se encaixa na classificação como contato com exposição a doença transmissível não especificada , com código internacional de doenças Z20.9. Assim sendo, tem o respaldo do órgão trabalhista que o assiste, e a instituição para qual presta serviço, tem a responsabilidade de realizar exames e fazer o acompanhamento.

O acidente de trabalho por material biológico é considerado uma emergência médica ${ }^{8}$, além de configurarse como um evento súbito, o acidente de trabalho é o acontecimento ocorrido no exercício de atividades laborais, independentemente da situação empregatícia e previdenciária do trabalhador acometido.

A preocupação com a biossegurança para os trabalhadores da área da saúde surgiu com o advento do HIV no meado dos anos 80 , fazendo com que fossem criadas normas de segurança frente a eventos biológicos e alavancando os estudos pertinentes a esta área. Em 1996, o Centers for Disease Control and Prevention (CDC) estabeleceu as precauções universais, de modo a serem utilizadas, independente da patologia ou sorologia do cliente $^{9-10}$.

A literatura traz a probabilidade de adquirir HIV, de acordo com o CDC, por infecção percutânea de $0,3 \%$, entretanto, o risco de infecção pelo vírus da hepatite $\mathrm{B}$ é de $30 \% \%^{6-11}$.

Mesmo com o conhecimento dos riscos torna-se indispensável o controle e medidas de proteção pré e pós-exposição, sendo a precaução padrão uma importante medida de prevenção. A imunização também ocupa um papel importante na prevenção da transmissão da hepatite $B$, porém, algumas classes funcionais mostram uma adesão insatisfatória ${ }^{12}$

Sabe-se dos riscos que envolvem o trabalhador que lida diariamente com pacientes. Entre estes riscos existe o biológico, e com isso, o de contágio de doenças através de acidentes com exposição por material biológico.Demonstrase que os acidentes com perfurocortantes destacam-se como a tipologia mais significativa na exposição biológica ocupacional. Os acidentes com objetos perfurocortantes ou de contato entre mucosas envolvendo material biológico, como sangue, tecidos ou outro material potencialmente infectado, estão relacionados à transmissão de patógenos virais como HIV, hepatite B ou $\mathrm{C}^{6}$.

Como conduta pós-exposição ao acidente de trabalho por material biológico deve-se observar a importância de um atendimento especializado, de modo que se avalie o acidente, sua gravidade e coleta de exames necessários, além de quimioprofilaxia quando for necessário. Também emerge como fator importante o registro através da comunicação de acidente de trabalho, e a notificação por acidente de trabalho com material biológico.

Algumas literaturas trazem que alguns profissionais não realizam medidas de profilaxia e realização de exames ou notificação quando sofrem este tipo de acidente, o que gera um número significativo de subnotificação para o Sistema Nacional de Agravos Notificáveis (SINAN) ${ }^{13}$.

A comunicação de acidente de trabalho é realizada para a Previdência Social, enquanto a notificação do SINAN é realizada para o Ministério da Saúde, na mesma plataforma informatizada onde são lançados os agravos notificáveis.

A importância deste registro está amparada nas necessidades dos gestores das instituições relacionar todos os fatores implicados na ocorrência dos acidentes, verificar os motivos mais frequentes, buscar soluções baseadas nas informações contidas no registro, implementar ações corretivas e avaliar a eficácia das mesmas. Entretanto, estudos demonstram que muitos incidentes com material biológico são subnotificados ${ }^{13}$.

A utilização das notificações visa realizar um diagnóstico precoce sobre eventos e acontecimentos à saúde da população, dando embasamento para explicar as causas dos agravos notificáveis, indicando as áreas mais críticas, fazendo com que a realidade epidemiológica da região seja conhecida9-14.

As notificações se apresentam como poderosa ferramenta para sinalizar epidemiologicamente os locais e agravos que estão emergindo no município do notificador, mostrando em que locais as ações de prevenção e promoção à saúde devem ser direcionados, a que tipo de público e a quais agravos especificamente.

Entretanto, mesmo ressaltando a importância do registro dos acidentes de trabalho por material biológico para o SINAN, muitos eventos ainda deixam de ser notificados, ou seja, resultam em subnotificações. Estas acabam influenciando na falha de análise epidemiológica em relação ao número de acidentes, tipos, principais agentes causadores, unidades onde mais ocorrem os agravos e as medidas que foram tomadas.

A Portaria $n^{\circ} 777 / G M$ do MS trata dos procedimentos técnicos para a notificação compulsória de agravos à saúde do trabalhador, onde o acidente de trabalho por material biológico está incluso. A regulamentação da notificação destes agravos deve ser efetuada em ficha própria, padronizada pelo Ministério da Saúde, no SINAN14.

Um estudo que foi realizado no município de São Paulo com notificações do SINAN NET, mostrou a existência de subnotificação nos estabelecimentos de saúde, tendo em vista a comparação com os profissionais expostos e o número de registros no SINAN ${ }^{15}$. Um fator que segundo a literatura contribui para o número de subnotificações é a pouca importância dada às pequenas lesões, ou o desconhecimento dos fluxos de encaminhamento do profissional pós-acidentado ${ }^{16}$. 
Outros estudos concluíram que a subnotificação acaba impactando nas ações de prevenção, ressaltando a importância da conscientização dos trabalhadores quanto à importância do registro de seus acidentes, de modo a abastecer adequadamente o sistema de informação em saúde e resguardá-lo quanto aos seus direitos como profissional da saúde, exposto a patógenos potencialmente infectantes.

Cabe ressaltar a importância da conscientização, informação, sensibilização, treinamento, fiscalização e disponibilização dos recursos materiais, principalmente dos dispositivos com sistema de segurança para evitar acidentes por material biológico ${ }^{16}$. Vale salientar também a importância de realizar efetivamente as notificações dos eventos ocorridos, pois as informações levantadas, relativas ao acidente, possibilitam identificar causas e gerir intervenções relacionadas a ele.

Aproximadamente $40 \%$ dos indivíduos que se acidentam por material biológico não cumprem as medidas de pós-exposição, não realizando exames sorológicos e notificação ou confecção da Comunicação de Acidente de Trabalho $(C A T)^{15-17}$. Sendo a relevância deste fator baseada nos riscos e prejuízos que os trabalhadores estão submetidos, ao ônus psicológico, humano e físico, e ao aumento de gastos para as instituições governamentais; além do fato de onerar a assistência previdenciária, pois os acidentes de trabalho que provocam lesão corporal, perda ou capacidade para o trabalho permanente ou temporária, uma vez que vários destes segurados têm a possibilidade de serem licenciados pela previdência social.

$\mathrm{Na}$ contramão desta importância estão os dados, encontrados em estudos, que mostram que apenas $29 \%$ das pessoas tiveram a emissão da CAT realizada ${ }^{6 \$ 15-19}$ Não bastasse, de se notar ainda as notificações que são realizadas de forma errônea ou incompleta, com informações insuficientes, falta de contato do trabalhador, ausência de exames laboratoriais, ocupação e vínculo empregatício; informações que são importantes para a caracterização do acidente e acidentado.

Em um estudo com odontólogos, mostrou-se que $50 \%$ das fichas de notificação não tinham as informações completas, impedindo assim a análise criteriosa dos acidentes, sugerindo que outros acidentes ocorriam sem que fosse realizado o registro apropriado ${ }^{20}$.

A limitação de informações acerca dos eventos biológicos que acometem acidentalmente os trabalhadores da área de saúde, especialmente os da enfermagem, como grupo que tem um quantitativo significante de funcionários nas instituições de saúde, mostra-se como uma falha no cumprimento dos fluxos estabelecidos pelo Ministério da Saúde, pois priva uma coleta fidedigna de dados epidemiológicos e emerge a ausência de uma cultura baseada em preocupações com direitos trabalhistas, fundamentada ainda na Política Nacional de Saúde do Trabalhador e Trabalhadora, onde mostra que deve-se realizar vigilância em saúde do trabalhador em todos os grupos, dando especial ênfase aos grupos de risco.
É evidente que os profissionais da área de saúde estão acondicionados em um grupo de risco, enfatizado pelo fato que no processo de seu trabalho está exposto a patógenos potencialmente contaminantes. Entre as ocupações, a enfermagem é a profissão mais acometida, com casos registrados de soroconversão e casos de infecção ocupacional ${ }^{21}$.

Alguns profissionais preferem omitir a ocorrência do acidente, pois imaginam que possam ocorrer julgamentos, tomando atitudes sem o respaldo de um profissional capacitado; medidas que poderiam definir o não aparecimento de um processo de adoecimento.

Estudos demonstram que alguns alunos sentemse receosos pela exposição acidental, e com medo de o acidente influenciar no julgamento e avaliação, de modo a não estarem aptos tecnicamente ${ }^{22}$. A prevenção e controle dos riscos ocupacionais emergem um campo que necessita ser estudado e expandido, pois as características do trabalho não devem ser critério para o aparecimento de doenças no homem6-10-20-23. Não se pode deixar de tratar da magnitude inserida na criação de um padrão para os acidentes. Através da análise retrospectiva dos acontecimentos, é possível oferecer intervenções eficazes.

As intervenções que minimizam os riscos de acidentes biológicos estão associadas ao treinamento e conhecimento dos profissionais acerca dos riscos, estabelecimento e implementação de fluxos, boas práticas embasadas na biossegurança, utilização de dispositivos com sistema de segurança e pró-atividade da equipe envolvida no processo de trabalho em querer auxiliar e internalizar que o risco é para todos que lidam diretamente com pacientes.

O risco apresenta-se maior para profissionais que lidam diretamente com pacientes, no exercício do cuidado e que não utilizam as normas de proteção universais: como o reencape de agulhas, ausência de luvas, entre outros ${ }^{24-25}$. No Brasil, existem poucos estudos na área de saúde do trabalhador que analisam a conduta sorológica dos profissionais acidentados no momento pós-acidente ${ }^{7}$.

Um município que se mostrou pioneiro, na visão relacionada à pós-exposição, foi Londrina, onde desde 1997 implementou-se o Protocolo de Atendimento de Acidentes por Material Biológico, que foi baseado no protocolo do Ministério da Saúde, que por sua vez adentrou a seara do acidente biológico no início da década de 90, através da Coordenação de Doenças Sexualmente Transmissíveis (DST) / A Síndrome da Imunodeficiência Adquirida (AIDS) ${ }^{26}$.

O Ministério da Saúde atualiza regularmente os protocolos relacionados às quimioprofilaxias e condutas pós-acidente, objetivando proporcionar as melhores condições de assistência à saúde dos trabalhadores, visando à assistência integral de suas necessidades.

Entre os eventos relacionados à saúde do trabalhador, que são lançados no Sistema de Informação de Agravos Notificáveis, destacam-se os acidentes de trabalho leve, grave e biológico. 0 número de notificações de outros 
agravos não é significativo. Assim sendo, um número expressivo dos eventos ocupacionais configura-se por dermatose ocupacional, entretanto, tal evento ocupa uma escala não significativa no quesito notificação ${ }^{27-29}$.

O SINAN ocupa o papel de sistema permanente para agravos notificáveis, apontado como principal ferramenta dos indicadores epidemiológicos, sendo os dados coletados na esfera municipal, transmitidos à esfera estadual e quinzenalmente enviados à esfera federal ${ }^{28-30}$. Alguns anos separam o inicio da implantação do SINAN e a data atual, porém, mesmo assim, ainda existem poucos estudos que correlacionam os casos e a implementação de medidas de prevenção específicas $^{31}$.

\section{Condutas de prevenção e após o acidente de trabalho por material biológico}

Os objetivos das precauções padrão são: proteger os profissionais de possíveis contaminações e prevenir infecções relacionadas à assistência em saúde ${ }^{32}$. Entretanto, ao adotar tais medidas o profissional diminui o risco de acidentes, porém, não o elimina.

$\mathrm{Na}$ ocorrência do acidente, onde o trabalhador se expõe a risco biológico, que vem a ser definido como: materiais que são derivados do processamento de diversos tipos de materiais que estão contaminados biologicamente e que podem de alguma maneira prejudicar a saúde do trabalhador. A maior parte dos eventos associados aos acidentes ocorre no momento da soroterapia, atividades que envolvem a manipulação constante de agulhas e scalps ${ }^{33}$.

Os riscos de transmissão de patógenos, em especial do vírus da AIDS e das hepatites B e C, presentes na assistência de saúde são bem conhecidos ${ }^{34}$. Entretanto, a insegurança, a falta de experiência, a ausência de habilidade técnica nos procedimentos e o nervosismo podem aumentar as chances do profissional ter acidentes ${ }^{35}$.

A Norma Regulamentadora (NR) 32 estabelece regras para o funcionamento dos serviços de saúde, com a finalidade de organizar e assegurar que as medidas de proteção e biossegurança sejam implementadas e respeitadas, valorizando e reiterando a utilização de vestimenta adequada, EPI, vacinação e dando outras disposições ${ }^{6-11-17-35-38}$.

Apesar de esforços para manter os trabalhadores longe dos acidentes biológicos serem relevantes, é importante o conhecimento das medidas que devem ser tomadas após o acidente. Deve-se colher sorologias de hepatite $\mathrm{B}$, hepatite $\mathrm{C}$ e HIV, tanto para o paciente fonte quanto para o trabalhador, preencher a CAT e a notificação de agravo notificável, indicar a quimioprofilaxia sempre que necessário e oferecer suporte emocional para o funcionário6-39-41

Sempre que for necessária a utilização, a profilaxia pós-exposição, conhecida como PPE, ou quimioprofilaxia, deverá ser feita de modo mais rápido possível, sendo iniciada no máximo em 72 horas, tendo duração de até 28 $\operatorname{dias}^{2-42-43}$

A busca por melhores condições no trabalho, assistência adequada e diminuição dos riscos no processo de trabalho, fazem parte de uma constante luta para os profissionais de saúde, que inúmeras vezes se expõem a condições insalubres, resultando em adoecimento ${ }^{44}$.

\section{CONCLUSÃO}

Os acidentes de trabalho com exposição a material biológico encontram-se como um importante problema de saúde ocupacional, pelo risco de contágio de doenças, necessidade de amparo psicológico do trabalhador acidentado e necessidade de cumprimento de fluxos corretos de pós-exposição ocupacional, incluindo a notificação para o Sistema Nacional de Agravos notificáveis. A estimulação de pesquisas na temática, visando o desenvolvimento de medidas de proteção individuais e coletivas, além da promoção da segurança nos ambientes de trabalho foram aspectos levantados nas publicações que embasaram este estudo.

A adoção de práticas seguras, a necessidade de cumprir as leis e normas regulamentadoras, já existentes, mostra a necessidade de fortalecer as práticas de educação continuada voltada para os aspectos da segurança ocupacional e boas práticas relacionadas a uma assistência de enfermagem.

Constata-se que as publicações científicas sobre os acidentes com materiais biológicos, voltados à promoção e orientação a saúde do trabalhador no Brasil ainda se mostram incipientes, conforme verificado nos estudos incluídos nesta revisão integrativa. Emerge assim, a necessidade de explorar, em toda a sua ampla dimensão, a segurança e cuidados com os trabalhadores de saúde que exercem sua prática laboral, expostos a diversos agentes, entre eles o biológico.

\section{REFERÊNCIAS}

1 Ribeiro RP, Martins JT, Marziale MHP, Robazzi MLCC. O adoecer pelo trabalho de enfermagem: uma revisão integrativa. Rev latinoam enferm [Internet]. 2012 [acesso em 14 jun 2015] ; 46(2):495-504. Disponível em: http://www.revistas.usp.br/ reeusp/article/view/40974

2 Garanhani ML, Martins JT, Robazzi MLCC et al. O trabalho de enfermagem em unidade de terapia intensiva: significados para técnicos de enfermagem. SMAD Rev eletrônica saúde mental alcool drog [Internet]. 2008 [acesso em 14 jun 2015]; 4(2):01-15. Disponível em: http://pepsic.bvsalud.org/scielo. php?script=sci_arttext\&pid=S1806-69762008000200007.

3 Jacques CC, Milanez B, Mattos RCO. Indicadores para centro de referência de saúde do trabalhador: proposição de um sistema de acompanhamento dos serviços de saúde. Ciênc saúde coletiva [Internet]. 2012 [acesso em 14 jun 2015]; 17(2):369-78. Disponível em: http://www.scielo.br/scielo. php?script=sci_arttext\&pid=S1413-81232012000200011. 
4 Kruse MHL, Cervelin, AF, Schenini FS, Ribeiro RG, Oliveira SG. Saúde e obesidade: discurso de enfermeiras. Aquichan. 2012; 109-21.

5 Schmidt DRC, Dantas RAS, Marziale MHP. Ansiedade e depressão entre profissionais de enfermagem que atuam em blocos cirúrgicos. Rev Esc Enferm USP [ Internet]. 2011[acesso em 14 jun 2015]; 45(2): 487-93. Disponível em: http://www. scielo.br/pdf/reeusp/v45n2/v45n2a25.pdf.

6 Medeiros EAS, Bakolswi E, Sassi SJG, Destra AS. Eventos adversos relacionados à profilaxia anti-retroviral em acidentes ocupacionais. Rev saúde pública [Internet]. 2007 [acesso em 14 jun 2015]; 41(2):294-92. Disponível em: http://www.readcube. com/articles/10.1590\%2FS0034-89102007000200018

7 Oliveira AC, Paiva MHR. Condutas pós acidente ocupacional por exposição a material biológico entre profissionais de serviço de urgência. Rev enferm UERJ [Internet]. 2014 jan-fev [acesso em 14 jun 2015]; 22(1):116-22. Disponível em: http:// www.scielo.br/pdf/reeusp/v43n3/a25v43n3.pdf

8 Paulino DCR, Lopes MVO, Rolim ILTP.Biossegurança e acidentes de trabalho com perfuros cortantes entre profissionais de enfermagem de Hospital universitário de Fortaleza. Cogitare enferm [Internet]. 2008 out-dez [acesso em 15 jun 2015]; 13(4):507-13. Disponível em: http://ojs.c3sl.ufpr.br/ojs2/ index.php/cogitare/article/view /13109 /8867

9 Silva TR, Rocha SA, Ayres JA, Juliani CMCM. Acidente com material perfurocortante entre profissionais de enfermagem de um hospital Universitário. Rev gaúch enferm [Internet]. 2010 dez [acesso em 14 jun 2015]; 31(4):615-22. Disponível em: http://www.scielo.br/pdf/rgenf/v31n4/a02v31n4.pdf.

10 Inga E, Lopez G, Kamyia C. Accidentes biológicos en estudiantes de medicina de una universidad peruana: prevalência,mecanismos y factores de riesgo. An Fac Med [Internet]. 2010. [acesso em 16 jun 2015]; 71(1):37-42. Disponível em: http://revistasinvestigacion.unmsm.edu.pe/ index.php/anales/article/view/70

11 Oliveira AC, Lopes ACS, Paiva MHRS. Acidentes ocupacionais por exposição a material biológico entre a equipe multiprofissional do atendimento pré hospitalar. Rev Esc Enferm USP [Internet]. 2009 [acesso em 16 jun 2015]; 43(3):677-83. Disponível em: http://www.scielo.br/scielo. php?pid=S0080-62342009000300025\&script=sci_arttext.

12 Lima LKOLL, Ana Clara FVT, Barros DX, Ferreira PS, Paiva EMM, Simões LLP. Acidentes com material biológico entre estudantes de odontologia no estado de Goiás e o papel das instituições de ensino. ROBRAC [Internet]. 2012 [acesso em 16 jun 2015]; 21(58):553-9. Disponível em: http://files.bvs.br/ upload/S/0104-7914/2012/v21n58/a3511.pdf

13 Ferreira MD, Pimenta FR, Facchin LT, Gir E,Canini SRMS. Subnotificação de acidentes biológicos pela enfermagem de um hospital Univesitário. Cienc enferm [Internet]. 2015 [acesso em 17 jun 2015]; 2:21-9. Disponível em: http://www. scielo.cl/pdf/cienf/v21n2/art_03.pdf

14 Calonga SMS. Análise dos fatores associados ao acidente de trabalho entre a equipe multiprofissional dos estabelecimentos de saúde do município de Aquidauana-MS [tese] [Internet]. Rio de Janeiro: Escola Nacional de Saúde Pública, Fundação Osvaldo Cruz, 2012. [acesso em 20 jun 2015]. Disponível em: bvssp.icict.fiocruz.br/lildbi/docsonline/ get.php?id=3353
15 Valim MD, Marziale MHP. Notificação de acidentes de trabalho com exposição a material biológico: estudo transversal. Online braz j nurs [Internet]. 2012 [acesso em 20 jun 2015]; 2(4). Disponível em: http://www.objnursing.uff.br/index.php/ nursing/article/viewFile/3537/pdf.

16 Junior AM,Quiaios A,Domingues JN, FerreiraA, Paixão S, Sá NLet al. Desfechos de acidente de trabalho com exposição a agente biológico. Rev bras crescimento desenvolv hum [Internet]. 2014 [acesso em 20 jun 2015]; 24(3):249-54. Disponível em: http://pepsic.bvsalud.org/scielo.php?pid=S0104$12822014000300003 \&$ script=sci_arttext \&tlng=pt.

17 Luize PB, Canini SRMS, Gir E, Toffano SEM. Condutas após exposição ocupacional a material biológico em um hospital especializado em oncologia. Texto \& contexto enferm [Internet]. 2015 Jan-Mar [acesso em 20 jun 2015]; 24(1):170-7. Disponível em: http://dx.doi.org/10.1590/010407072015002700013.

18 Chiodi MB, Marziale MHP, Robazzi MLCC. Acidentes de trabalho com material biológico entre trabalhadores de unidades de saúde pública. Rev latinoam enferm. [Internet]. 2007 jul-ago [acesso em 20 jun 2015]; 15(4):632-8. Disponível em: http://www.scielo.br/scielo. php?pid=S010411692007000400017\&script $=$ sci arttext\&tlng=pt.

19 Oliveira AC, Paiva MHRS. Análise dos acidentes ocupacionais com material biológico entre profissionais em serviços de atendimento pré-hospitalar. Rev latinoam enferm. [Internet] 2013 jan-fev [acesso em 12 abr 2015]; 21(1):309-15. Disponível em: http://www.scielo.br/scielo.php?pid=S0104$11692013000100004 \&$ script=sci_arttext\&tlng=pt .

20 Brozoski MA, Traina AA, Homem MGN, Deboni MCZ. Ocorrência de acidentes perfuro cortantes em um curso de odontologia. RGO [Internet]. 2010 jan- mar [acesso em 12 abr 2015]; 58(1):77-80. Disponível em: www.revistargo.com.br/include/ getdoc.php?id=4593\&article=904.

21 Soerensen AA, Moryia TM, Hayashida M, Robazzi MLCC. Acidentes com material biológico em profissionais do atendimento pré-hospitalar móvel. Rev enferm UERJ [Internet]. 2009 abr- jun [acesso em 20 jun 2015]; 17(2):2349. Disponível em: http://www.facenf.uerj.br/v17n2/ v17n2a17.pdf

22 Canalli RTC, Moryia TM, Hayashida M. Acidentes com material biológico entre estudantes de enfermagem. Rev enferm UERJ [Internet]. 2010 abr- jun [acesso em 25 abr 2015]; 18(2):25964. Disponível em: http://www.facenf.uerj.br/v18n2/ v18n2a16.pdf.

23 - Valim MD, Marziale MHP. Avaliação da exposição ocupacional a material biológico em serviços de saúde. Texto \& contexto enferm [Internet]. 2011 [acesso em 22 abr 2015]; 20(spe):13846. Disponível em: https://www.researchgate.net/profile/ Marilia_Valim/publication/237039242_Avaliacao_da_ exposicao_ocupacional_a_material_biologico_em_servicos de_saude/links/00b7d5324e4747d211000000.pdf

24 Santos SS, Costa NA, Mascarenhas MDM. Caracterização das exposições ocupacionais a material biológico entre trabalhadores de hospitais no município de Teresina, Piauí, 2007 a 2011. Epidemiol serv saúde [Internet]. 2013 jan-mar [acesso em 24 abr 2015]; 22(1):165-70. Disponível em: scielo. iec.pa.gov.br /pdf/ess /v22n1 /v22n1a17.pdf. 
25 Giacotti GM, Haeffner R, Solheid NLS, Miranda FMA, Sarquis LMM. Caracterização das vítimas e dos acidentes de trabalho com material biológico atendidas em um hospital público do Paraná, 2012. Epidemil serv saúde [Internet]. 2014 abrjun [acesso em 24 abr 2015]; 23(2):337-46. Disponível em: http://www.scielosp.org/ pdf/ress/v23n2/1679-4974ress-23-02-00337.pdf.

26 Spagnuolo RS, Baldo RCS, Guerrini IA. Análise epidemiológica dos acidentes com material biológico registrados no Centro de Referência em Saúde do Trabalhador - Londrina-PR. Rev bras epidemiol [Internet]. 2008 [acesso em 04 mai 2015]; 11(2):315-23. Disponível em: http://www.scielo.br/scielo. php?script=sci_arttext \&pid.

27 Lima LM, Oliveira CC, Rodrigues KMR. Exposição ocupacional por material biológico no hospital Santa Casa de Pelotas-2004 a 2008. Esc Anna Nery Rev Enferm [Internet]. 2011 jan- mar [acesso em 14 mai 2015]; 15(1):96-102. Disponível em: http://www.scielo.br/scielo. php?pid=s141481452011000100014\&script=sci_arttext

28 Garcia LP, Blank VLG. Condutas pós-exposição ocupacional a material biológico na odontologia. Rev saúde pública [Internet]. 2008 [acesso em 18 mar 2015]; 42(2):279-86. Disponível em: http://www.scielo.br/scielo.php?script=sci_ arttext \&pid=S0034-89102008000200013.

29 Galdino A, Santana VS, Ferrite S. Os centros de referência em saúde do trabalhador e a notificação de acidente de trabalho no Brasil. Cad saúde pública [Internet]. 2012 [acesso em 14 mai 2015]; 28(1):145-59. Disponível em: http:// www.scielo.br/scielo.php?script=sci_arttext \&pid=S0102311X2012000100015.

30 Silva AICD. Análise da qualidade dos registros de acidentes biológicos com matérias perfurocortantes na Fundação Osvaldo Cruz no período de 1999 a 2004. [tese] [internet]. Rio de Janeiro: Escola Nacional de Saúde Pública, Fundação Osvaldo Cruz, 2006. [acesso em 10 mai 2015]. Disponível em: http://www.arca.fiocruz.br/handle/icict/5192.

31 Julio RS, Filardi MBS, Marziale MHP. Acidentes de trabalho com material biológico ocorridos em municípios de Minas Gerais. Revista Brasileira de Enfermagem [Internet]. 2014 janfev [acesso em 04 jun 2015]; 67(1):119-26. Disponível em: www.scielo.br/scielo.php?script=sci_arttext\&pid=S0034.

32 Valim MD, Marziale MHP,Hayashida M, Martinez MR. Ocorrência de acidentes de trabalho com material biológico potencialmente contaminado em enfermeiros. Acta Paulista de Enfermagem [Internet]. 2014 [acesso em 17 abr 2015]; 27(3):280-6. Disponível em: www.scielo.br/pdf/ape/ v27n3/1982-0194-ape-027-003-0280.pdf.

33 Silva MR, Cortez EA, Valente GSC. Acidentes com materiais perfurocortantes e biológicos no ambiente hospitalar: análise de exposição ao risco e medidas preventivas. Rev 0 cuidado é funda [Internet]. 2011 abr-jun [acesso em 30 mar 2015]; 3(2):1856-72. Disponível em: http://www.seer.unirio.br/index. php/cuidado fundamental/ article/view/1280.

34 Sonder GJ, Regez RN, Brinkman K, Prin JM, Mulder JW. Prophylaxis and follow-up after possible exposure to hiv, hepatites B virus, and hepatitis C outside hospital: evaluation 2000 -3. Quality improvement report. BMJ [Internet]. $2005 \mathrm{abr}$ [acesso em 03 mai 2015]; 330:825-9. Disponível em: http:// www.bmj.com.
35 Tissot F, Erard V, Dang T, Cavassini M. Nonoccupational HIV post-exposure prophylaxis: a 10-year retrospective analysis. Hiv Medicine [Internet]. 2010 nov [acesso em 12 abr 2015]; 11:584-592. Disponível em: http://onlinelibrary.wiley.com/ doi/10.1111/j.1468-1293.2010.00826.x/full.

36 Machado JMH, Santana VS, Campos A, Ferrite S, Peres MC, Galdino A et al. Situação da Rede Nacional de Atenção Integral em Saúde do Trabalhador (Renast) no Brasil, 2008-2009. Rev bras saúde ocup [Internet]. 2012 jan [acesso em 03 jun 2015]; 38(128): 243-256. Disponível em: http://www.repositorio. ufba.br:8080/ri/bitstream/ri/18881/1/Renast.pdf.

37 Soares LG, Maques LM, Feli VE. Multicausalidade nos acidentes biológicos. Rev bras enferm [Internet]. 2013 nov-dez [acesso em 30 mar 2015]; 66(6): 854-9. Disponível em: http://www.scielo.br/scielo.php?script=sci_ arttext \&pid=S0034-71672013000600007.

38 Julio RS, Filardi MB,Marziale MH. Acidentes de trabalho com material biológico ocorrido em municípios de Minas Gerais. Rev bras enferm [Internet]. 2014 jan-fev [acesso em 30 mar 2015]; 67(1): 119-26. Disponível em: http://www.scielo.br/scielo. php?script=sci_arttext \&pid=S0034-71672014000100119.

39 Pimenta FR, Ferreira MD, Gir E, Hayashida M. Atendimento e seguimento clínico especializado de profissionais de enfermagem acidentados com material biológico. Rev Esc Enferm USP [Internet]. 2013 [acesso em 03 mai 2015]; 47(1):198-204. Disponível em: http://www.scielo.br/scielo. php?script=sci_arttext\&pid=S0080-62342013000100025.

40 Marziale MHP, Rocha FLR, Robazzi MLCC, Cenzi CM, Santos HEC, Tronvo MEM. Influência organizacional na ocorrência de acidentes de trabalho com exposição a material biológico. Rev latinoam enferm [Internet]. jan-fev 2013. [acesso em 05 jul 2015]; 21(Spec):[08 telas]. Disponível em: http://www. revistas.usp.br/rlae/article/view/52943/56944.

41 Barros DX, Tipple AFV, Lima LKO, Souza ACS, Neves ZCP, Salgado TA. Análise de 10 anos de acidentes biológicos na equipe de enfermagem. Rev. Eletr. Enfermagem [Internet]. 2016 [acesso em 25 mar 2016]; 18:e1157. Disponível em: https://revistas.ufg.br/fen/article/view/35493/21259.

42 Cordeiro TSMC, Neto JMC, Cardoso MCB, Mattos AIS, Santos KOB, Araujo TM. Acidente de trabalho com material biológico: descrição dos casos na Bahia. Rev epidemiol controle infecç [Internet]. 2016 [acesso em 10 jul 2016]; 6(2). Disponível em: https://online.unisc.br/seer/index.php/epidemiologia/article/ view/6218/4741.

43 Silva SMS, Gomes IVMP, Anjos MS. Acidentes perfurocortante: conhecimento e uso de dispositivos de segurança. Revista. saúde.com [Internet]. 2016 jul [acesso em 10 jul 2016]; 12(2):522-7. Disponível em: http://www.uesb.br/revista/rsc/ ojs/index.php/rsc/article/view/353/366.

44 Moraes ALO, Santos ASA, Bernardes KO. Notificação dos acidentes de trabalho por exposição a material biológico entre trabalhadores da saúde. Revista Pesquisa em Fisioterapia [Internet]. 2016 mai [acesso em 10 jul 2016]; 6(2):133-47. Disponível em: https://www5.bahiana.edu.br/index.php/ fisioterapia/article/view/877. 